\title{
Relaxation of the structure of simple metal ion complexes in aqueous solutions at up to supercritical conditions
}

Robert A. Mayanovic

Missouri State University

Sumedha Jayanetti

Missouri State University

A J. Anderson

W A. Bassett

I M. Chou

Follow this and additional works at: https://bearworks.missouristate.edu/articles-cnas

\section{Recommended Citation}

Mayanovic, Robert A., Sumedha Jayanetti, Alan J. Anderson, William A. Bassett, and I-Ming Chou. "Relaxation of the structure of simple metal ion complexes in aqueous solutions at up to supercritical conditions." The Journal of chemical physics 118, no. 2 (2003): 719-727.

This article or document was made available through BearWorks, the institutional repository of Missouri State University. The work contained in it may be protected by copyright and require permission of the copyright holder for reuse or redistribution.

For more information, please contact BearWorks@library.missouristate.edu. 


\title{
Relaxation of the structure of simple metal ion complexes in aqueous solutions at up to supercritical conditions
}

\author{
Robert A. Mayanovic ${ }^{\mathrm{a})}$ and Sumedha Jayanetti ${ }^{\mathrm{b})}$ \\ Department of Physics, Astronomy and Materials Science, Southwest Missouri State University, \\ Springfield, Missouri 65804 \\ Alan J. Anderson \\ Department of Geology, St. Francis Xavier University, P.O. Box 5000, Antigonish, \\ Nova Scotia B2G 2W5, Canada \\ William A. Bassett \\ Department of Geological Sciences, Cornell University, Ithaca, New York 14853 \\ I-Ming Chou \\ MS 954, U.S. Geological Survey, Reston, Virginia 20192
}

(Received 14 June 2002; accepted 4 October 2002)

\begin{abstract}
Our previous x-ray absorption fine structure studies of aqueous solutions revealed relaxation of the structure of complexes of $\mathrm{Zn}^{2+}, \mathrm{Fe}^{2+}, \mathrm{La}^{3+}$, and $\mathrm{Yb}^{3+}$ ions with increasing temperature. These complexes in general exhibit reduction of cation-ligand bond lengths with increasing temperature due to hydrogen bond breaking and loss of water of solvation. Because this results in an overall lowering of the equilibrium state of the complex, we refer to the variation of the structure as relaxation. In the case of $\mathrm{M}\left(\mathrm{H}_{2} \mathrm{O}\right)_{m}^{n+}(m=6-9$ at room temperature, $n=2,3)$ aquo ion complexes $(\mathrm{M}: \mathrm{Zn}, \mathrm{Fe}, \mathrm{La}, \mathrm{Yb})$, there is a similar reduction in the number of coordinating water molecules with temperature. The relaxation of the structure is shown to result in a lowering of the binding energy per cation-ligand pair of a complex with increasing temperature. A comparison of the rate of structure relaxation with temperature, which is framed in the context of volume thermoelastic constriction, shows this quantity to have a gradual and direct dependence on the difference in Pauling's electronegativity of the cation-ligand pairs making up the ion complex.

(C) 2003 American Institute of Physics. [DOI: 10.1063/1.1524616]
\end{abstract}

\section{INTRODUCTION}

The association of solvated ions in heated aqueous solutions under moderate pressures plays an important role in many physical processes. The solvation properties of water undergo significant changes with increasing temperatures $(T)$ and pressures $(P)$ as a result of hydrogen bond breaking. Consequently, the screening of solvated ions becomes less effective resulting in stronger ion-ion interactions under elevated $P-T$ conditions. This has the effect that both the speciation and the structure of metal ion complexes become altered to varying degrees in going from ambient to supercritical conditions in electrolyte solutions. A detailed theory of ion association and formation of complexes in aqueous solutions applicable for a broad range of concentrations and at up to supercritical conditions has challenged theorists since the early work of Born ${ }^{1}$ and of Debye and Huckel. ${ }^{2}$ The paucity of structure data for ion complexes in aqueous solutions at elevated $P-T$ conditions has been a major barrier to an improvement of our understanding of electrolyte solutions. X-ray absorption fine structure (XAFS) spectroscopy is proving to be useful for the study of structure of ion

\footnotetext{
a) Author to whom correspondence should be addressed. Fax: (417)-8366226; Electronic mail: ram051f@smsu.edu

b) On leave from the Department of Physics, University of Colombo, Colombo 3, Sri Lanka.
}

complexes in solutions up to supercritical conditions. Recent XAFS studies of various ions in aqueous solutions show variation of cation-ligand bond lengths, often coupled with other structure changes, with increasing temperature. ${ }^{3-15}$ Here we discuss the variations of the structure of several metal ion complexes with temperature based on observations from our XAFS studies ${ }^{3-9,16,17}$ in the hope that it will stimulate the development of either first-principles theory or molecular dynamics simulations that might adequately describe these results.

We consider in detail the trends in structure changes of $\mathrm{Zn}^{2+}, \mathrm{Fe}^{2+}, \mathrm{La}^{3+}$, and $\mathrm{Yb}^{3+}$ ion complexes as a function of temperature, which have been derived from our previously reported studies. ${ }^{3-9,16,17}$ The first-row transition metal (FTM) ions offer an interesting comparison to the lanthanide (Ln) ions. The chemical and physical characteristics of FTM ion complexes are largely dictated by the interaction between the $3 d$ electrons of the valence shell of these elements and the ligands. Consequently, the cation-ligand interaction exhibits partially covalent character, especially in the case of ions such as $\mathrm{Zn}^{2+} \cdot{ }^{18}$ Conversely, the $4 f$ electrons of the Ln ions, being well shielded by intervening electron shells, are largely nonreactive with neighboring ligands. In this sense, the lanthanides are thought to behave similarly to the alkali metals and interact with ligands in the formation of complexes mainly through electrostatic forces. ${ }^{19}$ An objective of ours 
TABLE I. Experimental parameters used to measure structure data of cation complexes from fluid samples during our previous investigations.

\begin{tabular}{|c|c|c|c|c|c|}
\hline Label Sample (aqueous) & $\begin{array}{l}\text { Absorption } \\
\text { edge }(\mathrm{eV})\end{array}$ & $\begin{array}{l}\text { Temp. } \\
\text { range } \\
\left({ }^{\circ} \mathrm{C}\right)\end{array}$ & $\begin{array}{c}\text { Max. } \\
\text { pressure } \\
(\mathrm{MPa})\end{array}$ & Complex type & Reference \\
\hline A Fluid inclusions & $\begin{array}{l}\text { Zn K (9659) } \\
\text { Fe K (7112) }\end{array}$ & $\begin{array}{l}25-430 \\
25-500\end{array}$ & - & $\begin{array}{l}\mathrm{ZnCl}_{4}^{2-} \\
\mathrm{Fe}\left(\mathrm{H}_{2} \mathrm{O}\right)_{6}^{2+}, \mathrm{FeCl}_{3}^{-}\end{array}$ & $3,4,5$ \\
\hline B $2 m \mathrm{ZnCl}_{2}$ & Zn K (9659) & $25-660$ & 800 & $\mathrm{Zn}\left(\mathrm{H}_{2} \mathrm{O}\right)_{2} \mathrm{Cl}_{2}$ & 6 \\
\hline C $1 m \mathrm{ZnCl}_{2} / 6 m \mathrm{NaCl}$ & Zn K (9659) & $25-495$ & 200 & $\mathrm{ZnCl}_{4}^{2-}$ & 6 \\
\hline D $1 m \mathrm{ZnBr}_{2} / 6 m \mathrm{NaBr}$ & $\begin{array}{l}\text { Zn K (9659) } \\
\operatorname{Br~K~(13474)~}\end{array}$ & $\begin{array}{l}25-500 \\
25-500\end{array}$ & $\begin{array}{l}130 \\
500\end{array}$ & $\mathrm{ZnBr}_{4}^{2-}$ & 7 \\
\hline E $0.007 m \mathrm{La} / 0.16 m \mathrm{HNO}_{3}$ & $\mathrm{La} \mathrm{L}_{3}(5483)$ & $25-300$ & 160 & $\mathrm{La}\left(\mathrm{H}_{2} \mathrm{O}\right)_{9}^{3+}$ & 8 \\
\hline $\mathrm{F} 0.006 m \mathrm{Yb} / 0.16 m \mathrm{HNO}_{3}$ & $\mathrm{Yb} \mathrm{L}_{3}(8944)$ & $25-500$ & 250 & $\mathrm{Yb}\left(\mathrm{H}_{2} \mathrm{O}\right)_{8}^{3+}$ & 9 \\
\hline $\mathrm{G} 0.006 m \mathrm{YbCl}_{3} / 0.017 m \mathrm{HCl}$ & $\mathrm{Yb} \mathrm{L}_{3}$ (8944) & $25-500$ & 270 & $\begin{array}{l}\mathrm{Yb}\left(\mathrm{H}_{2} \mathrm{O}\right)_{8}^{3+} \\
\mathrm{Yb}\left(\mathrm{H}_{2} \mathrm{O}\right)_{\delta-n} \mathrm{Cl}_{n}^{+3-n}(\delta \approx 7)\end{array}$ & 9 \\
\hline
\end{tabular}

has been to study trends in structure changes of simple inorganic ion complexes in aqueous solutions with variations in thermodynamic parameters and element type that may offer some clues to the nature of the cation-ligand interaction. Here, we present our observations on trends in structure characteristics of FTM and Ln complex ions with temperature. We discuss the significance of the temperaturedependent structure variations of the complexes to cationligand bonding.

\section{EXPERIMENT}

The XAFS measurements were made at the National Synchrotron Light Source (NSLS), Cornell High Energy Synchrotron Source (CHESS) and at the Advanced Photon Source (APS). Table I shows the solution or sample type previously investigated, the absorption edge of XAFS measurements, and range of $P-T$ conditions. XAFS spectra were measured from fluid inclusions in thin quartz wafers mounted on a Linkam THMS 600 heating stage. The fluorescence spectra were measured on the X26A microbeam line at the NSLS. ${ }^{3-5}$ The solution samples were loaded in a hydrothermal diamond anvil cell (HDAC). The cell was modified to minimize attenuation of $\mathrm{x}$-rays by laser milling the diamonds for the purpose of making XAFS measurement in either transmission or fluorescence mode. ${ }^{16,17}$ The HDAC has the capability for fluorescence XAFS measurements of dilute solutions at x-ray photon energies of $5300 \mathrm{eV}$ and below. For such measurements, the solution sample is sealed between the diamond anvils in a sample chamber consisting of a $300 \mu \mathrm{m}$ diameter cup-shaped cavity in the center of the upper diamond anvil face and a $300 \mu \mathrm{m}$ diameter hole in a $50 \mu \mathrm{m}$ thick Re gasket. Additional details about the fluorescence HDAC are given elsewhere., $8,9,16,17$ Transmission XAFS spectra were measured using gas ionization chambers. Fluorescence XAFS spectra were measured using either a thirteen-element Ge detector or a single-element $\mathrm{Si}(\mathrm{Li})$ detector. The data analysis procedures used to obtain the structure results from the XAFS spectra have been detailed elsewhere. $^{3-9}$

\section{DISCUSSION}

Figure 1 shows the variation of the cation-halide bond lengths with temperature for the first-row transition metal ion halide complexes. Figure 2 shows similar variations for cation-ligand distances for lanthanide aquo ion and chloro complexes. We reported from our previous studies that the $\mathrm{Zn}-\mathrm{Cl}^{6}$ and $\mathrm{Zn}-\mathrm{Br}^{7}$ bond length variations exhibit small but measurable nonlinear reduction with increasing temperature. Because these nonlinear trends are quite small and negligible, the rates of change of cation-ligand distances with temperature have been calculated using linear regression and are compiled in Table II. Provided we exclude La-O distances from consideration for the moment, the cation-ligand distances exhibit uniform reduction at a rate ranging from

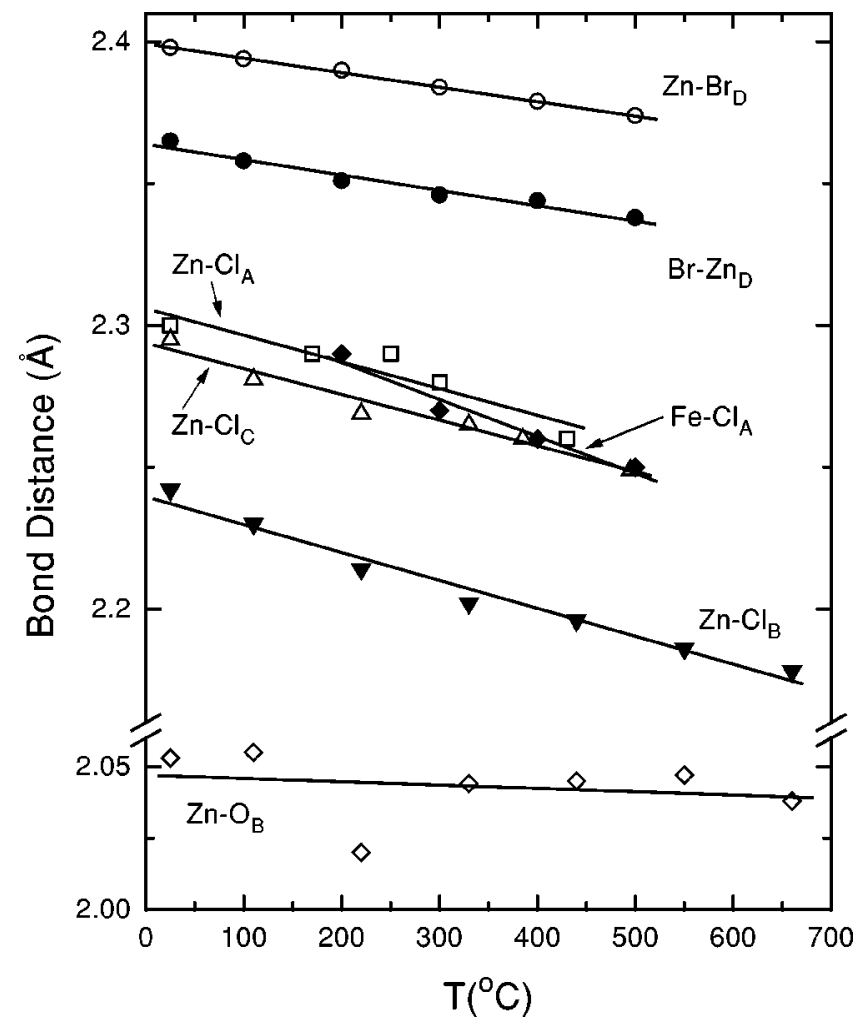

FIG. 1. The variation of the cation-ligand bond lengths as a function of temperature for the halide complexes of first-row transition metal ions $\mathrm{Fe}^{2+}$ and $\mathrm{Zn}^{2+}$. The first element of a cation-ligand pair indicates the photonabsorbing ion whereas the subscript indicates the solution sample referred to in Table I from which the XAFS was measured. The lines shown were obtained by linear regression fitting of the individual data sets for the cation-ligand pairs represented in the figure. 


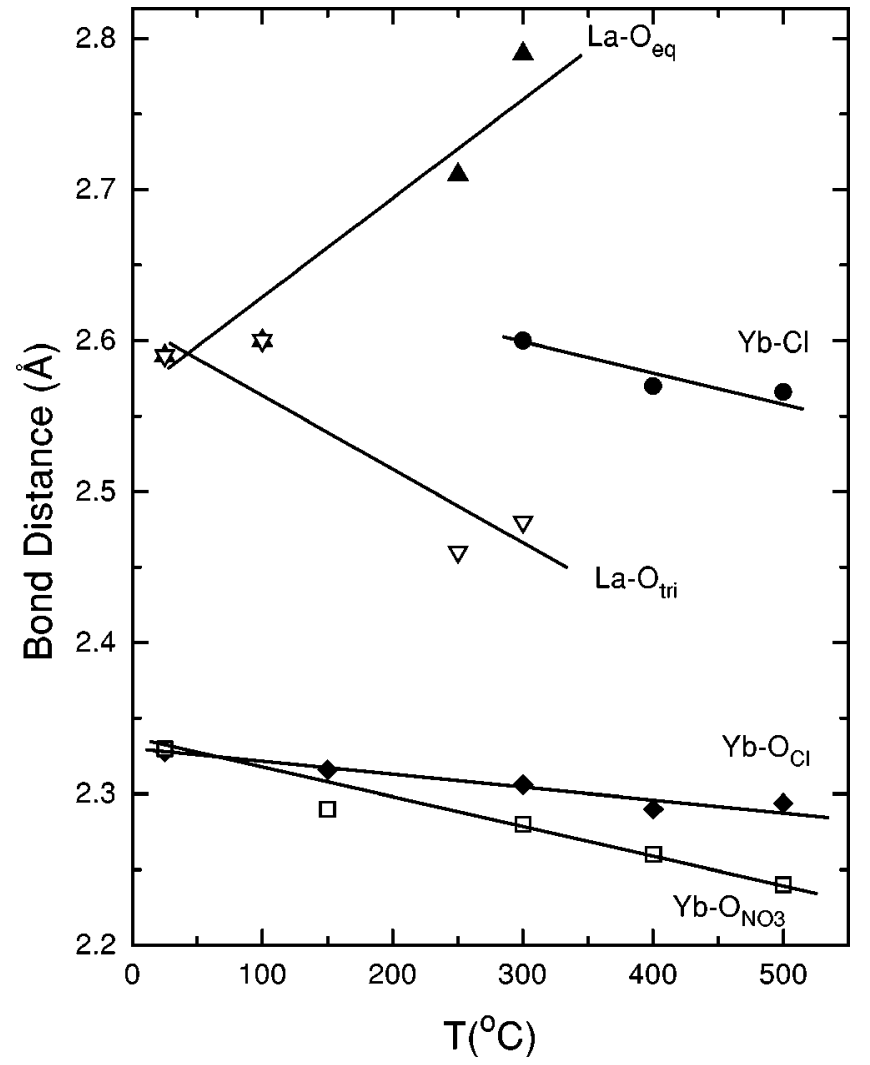

FIG. 2. The variation of the cation-ligand bond lengths as a function of temperature for the aquo ion and chloro complexes of lanthanide ions $\mathrm{La}^{3+}$ and $\mathrm{Yb}^{3+}$. The first element of a cation-ligand pair indicates the target ion and $\mathrm{Yb}-\mathrm{O}_{\mathrm{Cl}}$ and $\mathrm{Yb}-\mathrm{O}_{\mathrm{NO} 3}$ refer to ytterbium-oxygen bond lengths in $0.006 m \mathrm{YbCl}_{3} / 0.017 m \mathrm{HCl}$ and $0.006 m \mathrm{Yb} / 0.16 m \mathrm{HNO}_{3}$ solution samples, respectively, from which the XAFS was measured. $\mathrm{La}-\mathrm{O}_{\mathrm{eq}}$ and $\mathrm{La}-\mathrm{O}_{\text {tri }}$ represent the lanthanum-oxygen bond lengths to inner hydration shell water molecules in the equatorial and tricapped trigonal prismatic sites of the $\mathrm{La}^{3+}$ aquo ion in the $0.007 m \mathrm{La} / 0.16 m \mathrm{HNO}_{3}$ solution. The fit lines were obtained by linear regression of the individual data sets for the cation-ligand pairs represented in the figure.

$0.005 \AA / 100{ }^{\circ} \mathrm{C}$ to $0.017 \AA / 100{ }^{\circ} \mathrm{C}$, in their respective temperature ranges. In some cases, the reduction of cationligand bond lengths is accompanied by a reduction in the number of coordinated ligands with temperature. We discuss these effects in greater detail below.
There is a distinct difference between coordination number changes with temperature in the case of the halide complexes and of the aquo ions. The number of halide ligands in all cases either remains constant (in cases of a high halide:cation ratio) or increases with temperature. This occurs because solvation strength of water weakens, causing reduced screening of ions with temperature, leading to increased ion association and the formation of complexes or ion pairs. In terms of thermodynamic behavior of aqueous solutions, this can be directly attributed to the change of entropy dominating the change of enthalpy for ion association reactions under elevated temperatures. ${ }^{20}$ Conversely, the number of oxygen ligands (of hydration waters) either remains constant or decreases with temperature. A uniform reduction in the number of oxygen constituents of water molecules in the inner hydration shell of the $\mathrm{Yb}^{3+}$ aquo ion occurs with increasing temperature. The number of oxygen neighbors decreases from 8.3 to 4.8 in going from 25 to $500{ }^{\circ} \mathrm{C}$ (a $42 \%$ reduction). Similar results were found for various metal aquo ions from other XAFS studies made at elevated $P-T$ conditions. ${ }^{10-15}$

\section{A. Relaxation of the structure of first-row transition metal ion complexes in high $P-T$ aqueous solutions}

We first comment on some general features exhibited by our temperature-dependent structure results for the FTM of our studies. As shown in Fig. 1 and Table I, the slope $(\Delta R / \Delta T)$ of the $\mathrm{Zn}-\mathrm{Cl}$ bond length versus temperature is very consistent for all three samples (A, B and C) studied. The consistently larger values for $\mathrm{Zn}-\mathrm{Cl}$ distances for samples $\mathrm{A}$ and $\mathrm{C}$ in comparison to those measured in sample $\mathrm{B}$ are due to speciation differences. The predominant chloro complex in samples A and $\mathrm{C}$ is $\mathrm{ZnCl}_{4}^{2-}$ whereas in sample $\mathrm{C}$ the predominant complex is $\mathrm{Zn}\left(\mathrm{H}_{2} \mathrm{O}\right)_{2} \mathrm{Cl}_{2}$ throughout the $P-T$ range of measurement for the respective samples. We note that our room temperature $R_{\mathrm{Zn}-\mathrm{Cl}}$ values are in excellent agreement with prior results from large angle $\mathrm{x}$-ray scattering studies of various concentration zinc chloride aqueous solutions made by Maeda $e t$ al. ${ }^{21}$ and from x-ray diffraction measurements of $1 m \mathrm{ZnCl}_{2} / \mathrm{H}_{2} \mathrm{O}$ solution made by Paschina

TABLE II. Cation-ligand bond length relaxation characteristics for FTM and Ln ion complexes measured from samples identified in Table I. $\Delta R / \Delta T$ represents the slope of cation-ligand distance data shown as a function of temperature in Figs. 1 and $2, \bar{V}$ is the partial molal volume and $\Delta E N$ is the cation-ligand electronegativity difference.

\begin{tabular}{|c|c|c|c|c|c|}
\hline Sample & Complex & Bond & $\begin{array}{c}\Delta R / \Delta T \\
\left(10^{4} \AA /{ }^{\circ} \mathrm{C}\right)\end{array}$ & $\begin{array}{c}-\bar{V}_{0}^{-1}(\Delta \bar{V} / \Delta T) \\
\left(10^{4}{ }^{\circ} \mathrm{C}^{-1}\right)\end{array}$ & $\Delta E N$ (Pauling's) \\
\hline \multirow[t]{2}{*}{ A } & $\mathrm{ZnCl}_{4}^{2-}$ & $\mathrm{Zn}-\mathrm{Cl}$ & -0.95 & 1.24 & 1.51 \\
\hline & $\mathrm{FeCl}_{3}^{-}$ & $\mathrm{Fe}-\mathrm{Cl}$ & -1.3 & 1.69 & 1.33 \\
\hline \multirow[t]{2}{*}{$\mathrm{B}$} & $\mathrm{Zn}\left(\mathrm{H}_{2} \mathrm{O}\right)_{2} \mathrm{Cl}_{2}$ & $\mathrm{Zn}-\mathrm{Cl}$ & -1.0 & 1.34 & 1.51 \\
\hline & & $\mathrm{Zn}-\mathrm{O}$ & -0.25 & 0.37 & 1.79 \\
\hline $\mathrm{C}$ & $\mathrm{ZnCl}_{4}^{2-}$ & $\mathrm{Zn}-\mathrm{Cl}$ & -0.90 & 1.18 & 1.51 \\
\hline \multirow[t]{2}{*}{$\mathrm{D}$} & $\mathrm{ZnBr}_{4}^{2-}$ & $\mathrm{Zn}-\mathrm{Br}$ & -0.50 & 0.63 & 1.31 \\
\hline & & $\mathrm{Br}-\mathrm{Zn}$ & -0.50 & 0.63 & 1.31 \\
\hline \multirow[t]{2}{*}{$\mathrm{E}$} & $\mathrm{La}\left(\mathrm{H}_{2} \mathrm{O}\right)_{9}^{3+}$ & $\mathrm{La}-\mathrm{O}_{\text {eq. }}$ & $+7.1(-1.1)$ & $-8.22(1.27)$ & 2.34 \\
\hline & & $\mathrm{La}-\mathrm{O}_{\text {tri. }}$ & -5.2 & 6.02 & 2.34 \\
\hline $\mathrm{F}$ & $\mathrm{Yb}\left(\mathrm{H}_{2} \mathrm{O}\right)_{8}^{3+}$ & $\mathrm{Yb}-\mathrm{O}$ & -1.7 & 2.19 & 2.34 \\
\hline \multirow[t]{2}{*}{ G } & $\mathrm{Yb}\left(\mathrm{H}_{2} \mathrm{O}\right)_{8}^{3+}$ & $\mathrm{Yb}-\mathrm{O}$ & -0.80 & 1.03 & 2.34 \\
\hline & $\mathrm{Yb}\left(\mathrm{H}_{2} \mathrm{O}\right)_{\delta-n} \mathrm{Cl}_{n}^{+3-n}(\delta \approx 7)$ & $\mathrm{Yb}-\mathrm{Cl}$ & -1.7 & 1.93 & 2.06 \\
\hline
\end{tabular}


et $a l^{22}$ The $\mathrm{x}$-ray diffraction results for room temperature $R_{\mathrm{Zn}-\mathrm{Cl}}$ obtained by Kruh and Standley ${ }^{23}$ and XAFS results obtained by Drier and Rabe, ${ }^{24}$ from measurements of $\mathrm{ZnCl}_{2}$ aqueous solutions, are slightly larger $(0.04$ and $0.07 \AA$, respectively) but still within reason. The common slope $\Delta R / \Delta T$ of the two different chloro zinc complexes suggests that the relaxation of their structure is dependent primarily on the strength of the $\mathrm{Zn}-\mathrm{Cl}$ interaction.

As discussed in our prior work, ${ }^{7}$ the discrepancy in the $\mathrm{Zn}-\mathrm{Br}$ distance $\left(R_{\mathrm{Zn}-\mathrm{Br}}\right)$ of the $\mathrm{ZnBr}_{4}^{2-}$ complex measured from solution $\mathrm{D}(0.035 \AA)$ is most likely due to the inadequacy of FEFF8 in properly accounting for the onset of either the $\mathrm{Zn}$ or $\mathrm{Br} \mathrm{K}$ edge. Nevertheless, the roomtemperature $\mathrm{Zn}-\mathrm{Br}$ distance values measured from either $\mathrm{Zn}$ or Br K-edge XAFS spectra are in good agreement with results obtained from x-ray scattering measurements by Goggin et al. $^{25}$ and with XAFS results obtained by Lagarde et al. ${ }^{26}$ Note, however, that the slopes of the variation of $R_{\mathrm{Zn}-\mathrm{Br}}$ and of $R_{\mathrm{Br}-\mathrm{Zn}}$ with temperature are in excellent agreement.

The divalent state of iron in sample A was ascertained from a comparison of the near edge region of the Fe K-edge XAFS spectra measured from the fluid inclusions to those measured from ferrous and ferric sulfate powdered samples. There are no other comparable structure studies of iron(II) complexes in hydrothermal fluid samples. One of the first detailed concentration-dependent studies of ferric and ferrous complex structure and speciation was made by Apted et al. ${ }^{27}$ using XAFS measurements of saline aqueous solutions at room temperature. The authors ${ }^{27}$ determined that $\mathrm{Fe}\left(\mathrm{H}_{2} \mathrm{O}\right)_{6}^{2+}$ species predominate for all concentrations in the ferrous chloride solutions, in agreement with our results.

\section{B. Relaxation of the structure of Lanthanide ion complexes in high $P-T$ aqueous solutions}

We next turn our attention to examining the nature of variation of the structure of $\mathrm{Ln}^{3+}$ ion complexes in high $P-T$ aqueous solutions. As discussed in more detail below, the structure variation with temperature is exhibited in terms of either a bond contraction, or, as in the case of the $\mathrm{La}^{3+}$ aquo ion, a combination of bond contraction and expansion effects that occur in response to sudden changes in the stress field exerted by the solvating water molecules residing in the outer hydration shell. And, because this results in an overall lowering of the equilibrium state of the complex, we refer to the variation of the structure as relaxation.

The most striking features exhibited in Fig. 2 are the greater slopes for $\Delta R_{\mathrm{La}-\mathrm{O}_{\mathrm{eq}}} / \Delta T$ and $\Delta R_{\mathrm{La}-\mathrm{O}_{\mathrm{tri}}} / \Delta T$ than for other bond length temperature variations, where the equatorial $\left(O_{\text {eq }}\right)$ and tricapped trigonal prismatic $\left(O_{\text {tri }}\right)$ oxygen ligands of the inner hydration shell water molecules are represented thusly. As described by us previously, ${ }^{8,9}$ the relaxation exhibited by the $\mathrm{La}^{3+}$ aquo ion complex is notably different than for any other type of complex discussed here. Three oxygen neighbors in the equatorial plane of the $\mathrm{La}^{3+}$ aquo ion are shifted outwards by $0.12 \AA$, whereas six oxygen neighbors in the tricapped trigonal prismatic sites are contracted inwards by $0.13 \AA$, in going from 25 to $300^{\circ} \mathrm{C}$. No- tably, there is no reduction of the number of oxygen atoms of the $\mathrm{La}^{3+}$ aquo ion throughout the full temperature range.

It can be easily demonstrated using only ion-dipole electrostatic interactions that the net effect of relaxation of the $\mathrm{La}^{3+}$ aquo ion complex is energetically favorable. Accounting only for the ion-dipole interaction, we can express the energy of the hydrated $\mathrm{La}^{3+}$ ion as ${ }^{28}$

$$
E_{\mathrm{id}}=-\sum_{i=1}^{9} \mathbf{E}_{c}\left(\mathbf{r}_{i}\right) \cdot \mathbf{p}_{i}\left(\mathbf{r}_{i}\right)=-\sum_{i=1}^{9} \frac{q_{c}}{4 \pi \varepsilon_{0} r_{i}^{3}} \mathbf{r}_{i} \cdot \mathbf{p}_{i}\left(\mathbf{r}_{i}\right),
$$

where $\mathbf{E}_{c}$ is the point-charge electric field due to the cation, $\mathbf{r}_{i}$ is the position vector pointing from the cation to each of the water molecules of the inner hydration shell, $\mathbf{p}_{i}$ is the electric dipole moment of each of the water molecules, and $q_{c}$ is the charge of the cation $(+3 e)$. The electric dipole moment is expressed as a function of position relative to the cation to account for the polarizability of the water molecule under the presence of the cation's electric field. Assuming that the electric dipoles residing on the water molecules of the aquo ion complex are all oriented radially with respect to the cation, Eq. (1) simplifies accordingly,

$$
E_{\mathrm{id}}=-\frac{6 q_{c} p_{\mathrm{tri}}}{4 \pi \varepsilon_{0} r_{\mathrm{tri}}^{2}}-\frac{3 q_{c} p_{\mathrm{eq}}}{4 \pi \varepsilon_{0} r_{\mathrm{eq}}^{2}} .
$$

Here $p_{\text {tri }}$ and $p_{\text {eq }}$ represent the magnitudes of the electric dipole moments and $r_{\text {tri }}$ and $r_{\mathrm{eq}}$ the radial distances from $\mathrm{La}^{3+}$ to the oxygens of the water molecules residing in the tricapped trigonal prismatic and equatorial sites, respectively. We estimate the polarizability of each water molecule using the scaling function of Kowall et al., ${ }^{29}$

$$
S(r)=\frac{\left(R_{2}-r\right)^{2}\left(R_{2}+2 r-3 R_{1}\right)}{\left(R_{2}-R_{1}\right)^{3}},
$$

where $R_{2}=4.0 \AA$ and $R_{1}=2.1 \AA$ are the limiting radial distances for which $S$ results in 0 and 1, respectively, and $r$ is set equal to $r_{\text {tri }}, r_{\text {eq }}$, or $r_{0}$ for our calculations, where $r_{0}$ is the common $\mathrm{La}-\mathrm{O}$ radial distance at room temperature. We define $\alpha_{\text {tri }}=f \cdot\left(S\left(r_{\text {tri }}\right)-S\left(r_{0}\right)\right)$ and $\alpha_{\text {eq }}=f \cdot\left(S\left(r_{0}\right)-S\left(r_{\text {eq }}\right)\right)$ as the polarizabilities of the water molecules in the tricapped trigonal prismatic and equatorial sites, respectively. Because the scatter of $\mathrm{La}-\mathrm{O}$ bond lengths is appreciable (as reflected in the given error of $0.04 \AA$ ), we use the values interpolated from linear regression analysis for $r_{\text {tri }}=2.47 \AA$ and $r_{\text {eq }}$ $=2.77 \AA$. Furthermore, we use the value $f=0.3$ from results of Kowall et al., ${ }^{29}$ yielding 0.02 and 0.036 for $\alpha_{\text {tri }}$ and $\alpha_{\text {eq }}$, respectively.

Next, we express Eq. (2) in the following manner:

$$
\begin{aligned}
E_{\mathrm{id}}\left(300^{\circ} \mathrm{C}\right)= & -\frac{q_{c} p_{0}}{4 \pi \varepsilon_{0} r_{0}^{2}}\left[6 \frac{\left(1+\alpha_{\mathrm{tri}}\right)}{\left(1-\frac{\delta r_{\mathrm{tri}}}{r_{0}}\right)^{2}}\right. \\
& \left.+3 \frac{\left(1-\alpha_{\mathrm{eq}}\right)}{\left(1+\frac{\delta r_{\mathrm{eq}}}{r_{0}}\right)^{2}}\right]
\end{aligned}
$$

where $\delta r_{\text {tri }}=r_{0}-r_{\text {tri }}$ and $\delta r_{\text {eq }}=r_{\text {eq }}-r_{0}$ and $p_{0}$ represents the electric dipole moment (magnitude) of the water molecule in the first hydration shell of $\mathrm{La}^{3+}$ at room temperature. This 
can be expressed in terms of the ion-dipole interaction energy at room temperature $E_{\mathrm{id}, 0}=-9 \cdot\left(q_{c} p_{0}\right) /\left(4 \pi \varepsilon_{0} r_{0}^{2}\right)$,

$$
E_{\mathrm{id}}\left(300^{\circ} \mathrm{C}\right)=E_{\mathrm{id}, 0}\left[\frac{2\left(1+\alpha_{\mathrm{tri}}\right)}{3\left(1-\frac{\delta r_{\mathrm{tri}}}{r_{0}}\right)^{2}}+\frac{\left(1-\alpha_{\mathrm{eq}}\right)}{3\left(1+\frac{\delta r_{\mathrm{eq}}}{r_{0}}\right)^{2}}\right],
$$

yielding a value $E_{\mathrm{id}}\left(300^{\circ} \mathrm{C}\right)=E_{\mathrm{id}, 0}(1.03)$. Thus, we see that a $3 \%$ increase in the contribution to the magnitude of the binding energy from the ion-dipole interaction is realized from the relaxation of the $\mathrm{La}^{3+}$ aquo ion in going from room temperature to $300^{\circ} \mathrm{C}$. We find that the contribution to this increase in the absolute value of the binding energy from the polarizability of waters is significant $(\sim 19 \%)$. Note that this results in a lowering of the equilibrium state of the complex since the binding energy term $\left(E_{\text {id }}\right)$ becomes more negative. It can be shown quite directly that the dipole-dipole interaction energy and higher-order anisotropy terms of the hydrated and polarizable $\mathrm{La}^{3+}$ ion are negligible in comparison to its ion-dipole energy term and have therefore been omitted for the sake of clarity of discussion.

In order to see directly how the relaxation of the aquo ion complex leads to energy lowering, it is instructive to expand Eq. (5) to first order using the Binomial Theorem,

$$
\begin{aligned}
E_{\mathrm{id}}\left(300^{\circ} \mathrm{C}\right) \cong & E_{\mathrm{id}, 0}\left[\frac{2}{3}\left(1+\alpha_{\text {tri }}\right)\left(1+\frac{2 \delta_{\text {tri }}}{r_{0}}\right)\right. \\
& \left.+\frac{1}{3}\left(1-\alpha_{\mathrm{eq}}\right)\left(1-\frac{2 \delta r_{\mathrm{eq}}}{r_{0}}\right)\right] .
\end{aligned}
$$

Using the fact that $\delta r_{\text {eq }}=1.5 \delta r_{\text {tri }}$ at $300^{\circ} \mathrm{C}$, the above equation can be expressed in the following manner:

$$
\begin{aligned}
E_{\mathrm{id}}\left(300^{\circ} \mathrm{C}\right) \cong & E_{\mathrm{id}, 0}\left[1+\frac{\left(2 \alpha_{\mathrm{tri}}-\alpha_{\mathrm{eq}}\right)}{3}\right. \\
& \left.+\left(4 \alpha_{\mathrm{tri}}+3 \alpha_{\mathrm{eq}}+1\right) \frac{\delta r_{\mathrm{tri}}}{3 r_{0}}\right],
\end{aligned}
$$

from which we can see that the increase in ion-dipole energy is directly dependent upon the water molecule polarizability terms and the absolute value of the $\mathrm{La}-\mathrm{O}$ bond contraction. Note that Eq. (7) yields an increase in the iondipole contribution to the absolute value of the binding energy of $2 \%$, meaning that the first-order effects account for $\sim 70 \%$ of the lowering in $\mathrm{La}^{3+}-\mathrm{H}_{2} \mathrm{O}$ interaction energy. Furthermore, it can easily be shown that second-order terms of this type of expansion account for nearly the remaining $30 \%$. Thus, despite the complicated nature of the relaxation of the $\mathrm{La}^{3+}$ aquo ion complex, the net effect leads to a reduction of its binding energy. This is so because more of the water molecules (in trigonal sites) are displaced towards the $\mathrm{La}^{3+}$ ion than away from it (in equatorial sites). It is difficult to imagine how a uniform spherical solvent cavity beginning at the outer hydration shell, exerting uniform partial volume pressure, can trigger the anisotropic relaxation of the $\mathrm{La}^{3+}$ aquo ion complex. However, such uniformity is only preserved at the lowest $P-T$ conditions (close to ambient conditions). As we describe in greater detail below, hydrogen bond breaking and shedding of outer hydration shell water molecules at elevated $P-T$ points breaks the uniformity in the surrounding solvent, thus creating the conditions leading to contraction of the $\mathrm{La}-\mathrm{O}_{\text {tri }}$ bonds and lengthening of the $\mathrm{La}-\mathrm{O}_{\mathrm{eq}}$ bonds of the $\mathrm{La}^{3+}$ aquo ion complex.

It is easy to see that in cases where a simple contraction of the hydration shell occurs, such as in the case of the $\mathrm{Yb}^{3+}$ aquo ion complex, the relaxation of the structure under high $P-T$ conditions in aqueous solutions will lead to an overall lowering of the ion-dipole interaction energy. We note that using the procedures outlined above, the relaxation of the structure of the $\mathrm{Yb}^{3+}$ aquo ion complex leads to a lowering of its ion-dipole binding energy by $9 \%$ per water molecule in going from 25 to $500^{\circ} \mathrm{C}$. Nevertheless, there is a net energy increase that takes effect from the shedding of water molecules, from $\sim 8$ to $\sim 5$ in progressing through the same temperature range. Given that the ion-dipole attraction is the dominant cation-ligand interaction of hydrated $\mathrm{Ln}^{3+}$ ions, it is safe to conclude that relaxation of the structure results in the overall lowering (or increase in the magnitude) of the binding energy per $\mathrm{Ln}-\mathrm{H}_{2} \mathrm{O}$ pair.

\section{Volume thermoelastic constriction of ion complexes}

From our Br K-edge XAFS measurements made on a $1 m \mathrm{ZnBr}_{2} / 6 m \mathrm{NaBr}$ aqueous solution, we were able to ascertain the effect of anion-oxygen bond breaking on relaxation of the structure of ion complexes with temperature. A greater than $60 \%$ reduction in bromine-oxygen bonds for both the $\mathrm{ZnBr}_{4}^{2-}$ complex and the $\mathrm{Br}^{-}$aquo ion in going from $25^{\circ} \mathrm{C}$ to $500{ }^{\circ} \mathrm{C}$ was reported from our study. ${ }^{7}$ In addition, the results from our study showed that the bromine ions and the oxygen neighbors of the waters in the hydration shells (both inner and outer) are weakly hydrogen bonded. We attribute the relaxation of the structure of ion complexes directly to progressive hydrogen bond breaking and commensurate reduction of the outer-hydration shell water molecules in going from ambient to supercritical conditions in the aqueous solutions.

We infer from our results that the relaxation mechanism occurs due to unbalanced cation-ligand interaction forces causing contraction of the ligands inward toward the cation. This is shown schematically in Fig. 3. At low temperatures while the outer shell is intact, each ligand of a given complex has a balance of forces between the cation-ligand and ligand-water-molecules (in the outer shell) interactions. As the hydrogen bonds between the ligands and the outerhydration shell oxygen atoms of the water molecules become broken at elevated temperatures, an imbalance toward the cation-ligand bond results allowing the ligand to relax by shifting inward toward the cation of the ion complex. Note that bond contraction also occurs in this simple model at the ligand sites where outer-hydration-shell water molecules remain bonded, as a result of the disruption of the net force balance and of the symmetric stress field on the ion complex occurring under ambient conditions. Alternatively, bond contraction of simple ion complexes can also be understood on the basis of the conservation of energy. Given that hydrogen bond breaking occurring in the outer hydration shell results in the elevation of the energy of the ion complex, local equi- 

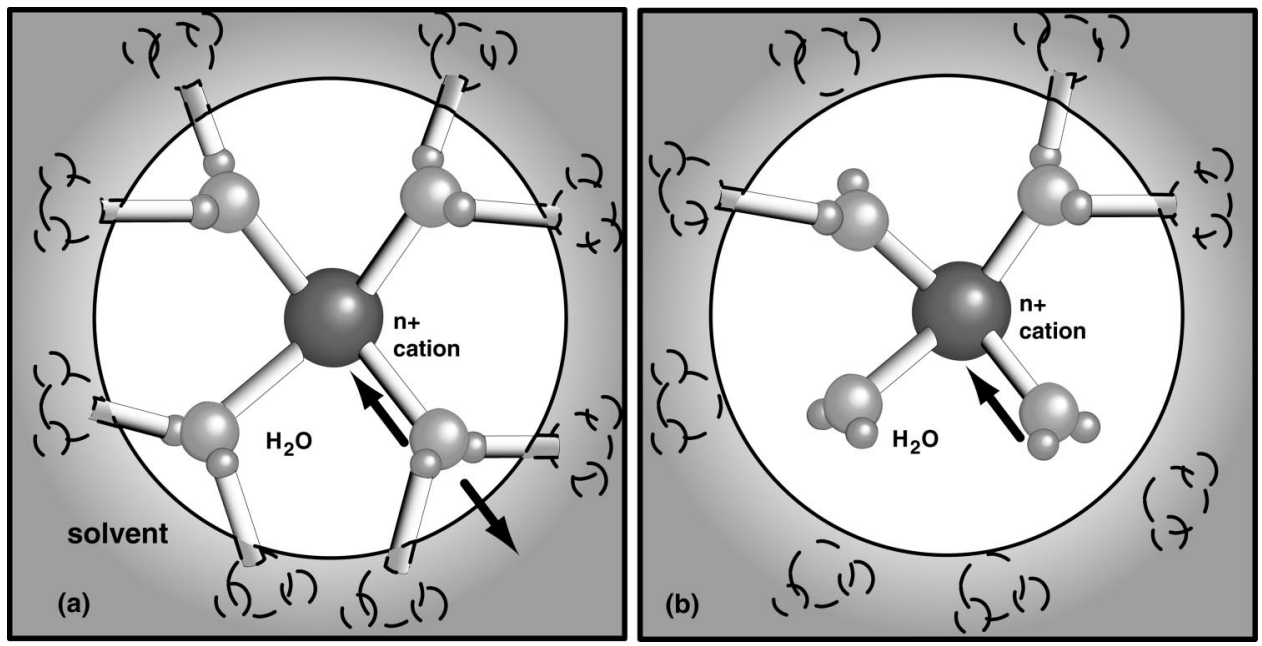

FIG. 3. A schematic figure demonstrating the proposed mechanism responsible for the relaxation of the structure of ion complexes in aqueous fluids under elevated $P-T$ conditions. (a) Under ambient conditions, the ligands (inner shell waters in diagram) are shown to be fully bonded to the surrounding solvent cavity containing the outer hydration shell. (b) Under near critical conditions, the hydrogen bonds to the surrounding solvent cavity are extensively broken resulting in imbalance of restoring forces acting on the complex. This generally leads to a contraction of the cation-ligand bonds at elevated $P-T$ conditions.

librium can be achieved through the (net) bond contraction mechanism. Because the entropy change results from bond breaking, this picture is fully consistent with the entropy change driving the ion association chemistry of a solution at high temperatures. ${ }^{20}$ Note that the relaxation of ion complexes contributes directly to the enthalpy of a given solution.

Given that it is possible to calculate by means of a simple electrostatic model the net ion-dipole interaction energy of the $\mathrm{La}^{3+}$ aquo ion complex, it follows that the net effect of displacement of the equatorial and tricapped trigonal prismatic oxygens at elevated temperatures can similarly be determined. Thus, we use $\Delta R_{\mathrm{La}-\mathrm{O}} / \Delta T=\frac{1}{3} \Delta R_{\mathrm{La}-\mathrm{O}_{\mathrm{eq}}} / \Delta T$ $+\frac{2}{3} \Delta R_{\mathrm{La}-\mathrm{O}_{\text {tri }}} / \Delta T$, where the slopes are weighted according to the proportions of equatorial and trigonal oxygens in the hydration shell, respectively. This value $(-1.1$ $\times 10^{-4} \AA /{ }^{\circ} \mathrm{C}$ ) is shown in Table II.

The relaxation of the ion complexes can be related to the variation of the partial molal volume $\bar{V}$ of the complex with temperature in the following manner. Using the partial molal volume $\bar{V}$ in the manner of Couture and Laidler ${ }^{30}$ and Hepler $^{31}$ we define the quantity

$$
K_{e}=-\frac{1}{\bar{V}}\left(\frac{\partial \bar{V}}{\partial T}\right)_{P}
$$

as the partial molal thermoelastic coefficient and approximate $\bar{V}$ in terms of a spherical volume of an ion complex. This quantity can be directly calculated from the bond contraction slope by the following expression:

$$
K_{e}=-\frac{3}{R_{0}}\left(\frac{\partial R}{\partial T}\right)_{P} \cong-\frac{3}{R_{0}}\left(\frac{\Delta R}{\Delta T}\right) .
$$

Here, $R_{0}$ represents the cation-ligand distance at room temperature. The values calculated using Eq. (9) are shown in Table II. We note that the condition of the pressure being held constant is well approximated in our experiments since our results bear negligible pressure dependence for the range of pressures of our experiments. ${ }^{3,6-8}$ For the $\mathrm{La}^{3+}$ aquo ion complex we show values of the partial molal thermoelastic coefficient $K_{e}$ for the $\mathrm{La}-\mathrm{O}_{\text {tri }}$ bonds and the $\mathrm{La}-\mathrm{O}_{\mathrm{eq}}$ bonds separately and the weighted value for overall $\mathrm{La}-\mathrm{O}$ relaxation in parenthesis.

We show in Fig. 4 values of the partial molal thermoelastic coefficient versus the difference in Pauling's electronegativity $(\triangle E N)$ for the cation-ligand pairs of each of the ion complexes. The most dramatic aspect of Fig. 4 is that it clearly shows a difference in the relaxation behavior of the FTM and Ln complexes. The FTM complexes all lie towards the smaller $\triangle E N$ side and in general have smaller partial molal thermoelastic coefficient values, whereas the converse is true for $\mathrm{Ln}$ complexes.

Despite scatter in the data, we observe a direct depen-

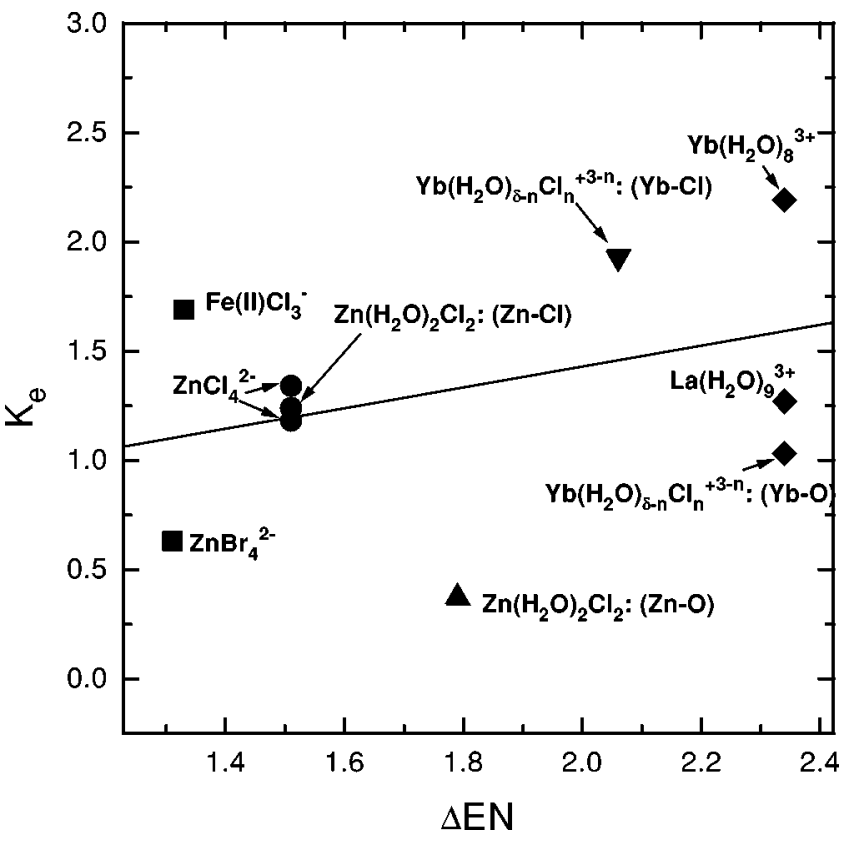

FIG. 4. The variation of the partial molal thermoelastic coefficient $K_{e}$ with the difference in Pauling's electronegativity $(\triangle E N)$ of the cation-ligand pairs making up the various ion complexes measured from our previous studies. Separate cation-ligand pairs are indicated in parenthesis beside each of the labels of mixed ligand complexes. The solid line indicates the linear regression fit to the data. 
dence of the degree of volume thermoelastic constriction on the difference in Pauling's electronegativity of the cationligand pairs making up the ion complex. This is reasonable since large values of $\Delta E N$ are associated with predominantly ionic character whereas small values imply a greater degree of covalent character of cation-ligand bonding. This can be attributed largely to the difference in character between the $4 f$ electrons of the Ln ions and $3 d$ electrons of the FTM ions. The $4 f$ electrons of the lanthanides, being well shielded by intervening electron shells, are largely nonreactive with neighboring ligands. In this sense, the lanthanides behave similarly to the alkaline earths and interact with ligands in the formation of complexes mainly through electrostatic forces. Conversely, the characteristics of FTM ions are largely determined by the interaction between the $3 d$ electrons of the valence shell of these elements and the ligands. Since covalent bonding is stronger than ionic bonding, FTM cation-ligand pairs having smaller $\Delta E N$ values are more strongly bonded than Ln cation-ligand pairs. Therefore, based on this simple picture, the ligands of FTM complexes should be displaced outwards to a smaller extent than the ligands of Ln complexes upon the formation of weak hydrogen bonds with waters of the surrounding solvent. Nevertheless, it should be borne in mind that a small degree of covalentlike character may be expected even for the interactions of Ln cation-ligand pairs. ${ }^{32}$ This and other details of cation-ligand interactions in hydrothermal solutions may in the future help explain some of our results, such as the scatter of data points shown in Fig. 4 and the anisotropic nature of the first hydration shell of $\mathrm{La}^{3+}$ ion at high temperatures.

The data point corresponding to $K_{e}$ of $\mathrm{Zn}-\mathrm{O}$ in the $\mathrm{Zn}\left(\mathrm{H}_{2} \mathrm{O}\right)_{2} \mathrm{Cl}_{2}$ complex deviates noticeably from the trend observed for the remaining points. For mixed-ligand and step-wise complexes of this type, we find noticeably greater uncertainties associated with structure results corresponding to oxygen coordination on account of its considerably weaker backscattering in comparison to that of chlorine. Thus, one explanation for the deviation might be simply due to considerably greater error associated with the $K_{e}$ data point of $\mathrm{Zn}-\mathrm{O}$. However, it seems also likely that the deviation reflects the added nature of attractive interaction of oxygens (of the water molecules) with the chlorine ions of the $\mathrm{Zn}\left(\mathrm{H}_{2} \mathrm{O}\right)_{2} \mathrm{Cl}_{2}$ complex. In this way, the inner-hydration shell oxygens may be more tightly bound in the $\mathrm{Zn}\left(\mathrm{H}_{2} \mathrm{O}\right)_{2} \mathrm{Cl}_{2}$ complex than in the $\mathrm{Zn}\left(\mathrm{H}_{2} \mathrm{O}\right)_{6}^{2-}$ aquo ion complex.

Although Eq. (8) coincides with the definition of the thermal expansion coefficient of materials, the two are not the same. Thermal expansion in solids for example, results from the ions occupying levels in the phonon density of states having an anharmonic character. In water, bulk thermal expansion is associated with hydrogen bond breaking within its network structure at high temperatures. Conversely, volume thermoelastic constriction evident in the relaxation of simple ion complexes results from a significant reduction in the coordination of the inner-sphere complex to the outer hydration shell of the solvent. Nevertheless, volume thermoelastic constriction of ion complexes is expected to have a slight retarding effect on thermal expansion of concentrated electrolyte solutions.

Furthermore, volume thermoelastic constriction of ionic complexes in electrolyte solutions is directly related to the partial molal adiabatic compressibility $\bar{\beta}_{S}$. Using thermodynamic network equations, ${ }^{33}$ one can arrive at an expression for $\bar{\beta}_{S}$,

$$
\bar{\beta}_{S}=-\frac{T}{\bar{V} \bar{C}_{P}}\left(\frac{\partial \bar{V}}{\partial T}\right)_{P}^{2}-\frac{1}{\bar{V}}\left(\frac{\partial \bar{V}}{\partial P}\right)_{T},
$$

where $\bar{C}_{P}$ is the partial molal constant-pressure heat capacity. The last term in Eq. (10) is the partial molal isothermal compressibility $\bar{\beta}_{T}$. We can recast Eq. (10) in the following manner:

$$
K_{e}^{2}=\frac{\bar{C}_{P}}{\bar{V} T}\left(\bar{\beta}_{T}-\bar{\beta}_{S}\right)
$$

Thus, we see that the square of the partial molal thermoelastic coefficient is related directly to the difference between the isothermal and adiabatic partial molal compressibilites of a complex. We can see by inspection of Eq. (11) that the partial molal isothermal compressibility of a complex species dominates the corresponding adiabatic quantity. This is reasonable if we postulate that its outer hydration shell has a direct bearing on the compressibility of an ion complex through electrostriction effects. Note that this is a more extended approach than electrostriction effects attributed to the primary or inner solvation shell of a complex. ${ }^{34}$ It has been observed that hydrogen bond breaking occurs with increasing pressure, although to a much lesser degree than with temperature. ${ }^{35}$ An increase in ambient pressure of the fluid under isothermal conditions will therefore result in greater disorder of the outer hydration shell of an ion complex and thereby a lessening of electrostriction. However, by its very nature, the outer hydration shell is expected to remain largely intact under adiabatic conditions. Thus, the compressibility of an ion complex is greater under isothermal conditions than under adiabatic conditions. We note that it may be possible under reduced densities and at sufficiently high temperatures to observe emerging rotational modes of an ion complex provided it is not coordinated (i.e., all hydrogen bonds are broken) to the outer hydration sphere. Provided such conditions are possible, the rotational modes could be detected using infrared spectroscopy.

\section{Comparisons to molecular dynamics and Monte Carlo simulations}

Molecular dynamics and Monte Carlo simulations offer an ideal bridge between experiment and theory because direct comparison to measurements of the microstructure of solutions can be made through calculation of radial distribution, correlation, local density, and other structure variables of a system. The challenge of making molecular dynamics simulations of solutions at elevated $P-T$ conditions often involves the incorporation of many-body effects, persisting on both the extended and local length scales, in the pair-wise particle interaction scheme employed in most calculations. 
Recent molecular dynamics simulations of association of $\mathrm{Cl}^{-}$and $\mathrm{Na}^{+}$(Ref. 36) and of $\mathrm{Cl}^{-}$and $\mathrm{H}_{3} \mathrm{O}^{+}$(Ref. 37) ions in high $P-T$ aqueous solutions have demonstrated a certain degree of success in modeling these systems. However, such approaches can give inaccurate results as evidenced from the molecular dynamics simulations of $\mathrm{SrCl}_{2}$ aqueous solutions at up to $527^{\circ} \mathrm{C} .{ }^{38}$ Although the correct contraction of the $\mathrm{Sr}-\mathrm{Cl}$ distance was shown from this study, the number of water molecules in the hydration shell of the $\mathrm{Cl}^{-}$anion was incorrectly predicted to increase with temperature. This contradicts most of the experimental evidence, including recent temperature-dependent XAFS results for $\mathrm{Br}^{-}$hydration reported by Wallen et al. ${ }^{13}$ and by Mayanovic et al. ${ }^{7}$ Furthermore, the $\mathrm{Cl}^{-}-\mathrm{H}_{2} \mathrm{O}$ distance to the first hydration shell was predicted to increase with increasing temperatures, which contradicts the results given by Wallen et al. ${ }^{13}$ and Mayanovic et al. ${ }^{7}$

In order to model accurately the solvation effects of FTM ion complexes and aqua ions by the surrounding water molecules under all $P-T$ conditions, ab-initio molecular dynamics simulations offer the most promising approach. ${ }^{39}$ In this case, first principles methods are employed to calculate the many-body interactions from the Schrödinger equation for the ion+water-molecules system. Lubin et al. have recently applied this method with considerable success to model the behavior of the $\mathrm{Al}^{3+}$ aquo ion up to $250^{\circ} \mathrm{C}$ and at equilibrium vapor pressures. ${ }^{40}$ Similar calculations carried out for hydrated $\mathrm{Fe}^{2+}$ and $\mathrm{Fe}^{3+}$ ions show correctly the changes in coordination in the hydration shell due to hydrolysis. ${ }^{41}$

\section{CONCLUSIONS}

We have shown that structure relaxation of $\mathrm{Zn}^{2+}, \mathrm{Fe}^{2+}$, $\mathrm{La}^{3+}$, and $\mathrm{Yb}^{3+}$ ion complexes leads to an overall lowering of their individual binding energies in going from ambient to high $P-T$ conditions in aqueous solutions. The structure relaxation, which takes the form of volume thermoelastic constriction of ion complexes, is due to hydrogen bond breaking and loss of water of solvation. The degree of structure relaxation can be quantified in terms of a partial molal thermoelastic coefficient, which is directly related to the rate of change in the cation-ligand distance with temperature. The partial molal thermoelastic coefficient has a gradual and direct dependence on the difference in Pauling's electronegativity of the cation-ligand pairs making up an ion complex. In relating this quantity to the partial molal volume, we find that its square is also directly proportional to the difference in the isothermal and adiabatic compressibilites of an ion complex. Furthermore, our results suggest that the concept of electrostriction of an ion complex should be extended to include the outer hydration shell in aqueous solutions.

\section{ACKNOWLEDGMENTS}

The authors wish to thank Steve Heald and others at the PNC-CAT beam line at the Advanced Photon Source (APS), Kenneth Finkelstein and other staff members at the Cornell High Energy Synchrotron Source (CHESS), and Steve Sutton and Mark Rivers for beam line development and others at X26A at the National Synchrotron Light Source (NSLS). This research was funded by a grant from the Research Corporation to R.A.M. and the Natural Sciences and Engineering Research Council of Canada (NSERC) grant to A.J.A. The U.S. Department of Energy (DOE), Basic Energy Sciences, Office of Science, under Contract No. W-31-109Eng-38 and DE-FG03-97ER45628 (PNC-CAT) supported the use of the APS. CHESS is supported by National Science Foundation Grant No. DMR97-13424. The NSLS was funded by DOE Grant No. DE-ACO2-76CH00016.

${ }^{1}$ M. Born, Z. Phys. 1, 45 (1920).

${ }^{2}$ P. Debye and E. Huckel, Z. Phys. 24, 305 (1923).

${ }^{3}$ R. A. Mayanovic, A. J. Anderson, and S. Bajt, Mater. Res. Soc. Symp. Proc. 437, 203 (1997).

${ }^{4}$ A. J. Anderson, R. A. Mayanovic, and S. Bajt, Can. Mineral. 36, 511 (1998).

${ }^{5}$ R. A. Mayanovic, A. J. Anderson, and S. Bajt, J. Phys. IV 7, C2-1029 (1997).

${ }^{6}$ R. A. Mayanovic, A. J. Anderson, W. A. Bassett, and I-M. Chou, J. Synchrotron Radiat. 6, 195 (1999).

${ }^{7}$ R. A. Mayanovic, A. J. Anderson, W. A. Bassett, and I-M. Chou, Chem. Phys. Lett. 336, 212 (2001)

${ }^{8}$ A. J. Anderson, S. Jayanetti, R. A. Mayanovic, W. A. Bassett, and I-M. Chou, Am. Mineral. 87, 262 (2002).

${ }^{9}$ R. A. Mayanovic, S. Jayanetti, A. J. Anderson, W. A. Bassett, and I-M. Chou, J. Phys. Chem. A 106, 6591 (2002).

${ }^{10}$ D. M. Pfund, J. G. Darab, J. L. Fulton, and Y. Ma, J. Phys. Chem. 98, 13102 (1994).

${ }^{11}$ S. L. Wallen, B. J. Palmer, and J. L. Fulton, J. Chem. Phys. 108, 4039 (1998).

${ }^{12}$ J. L. Fulton, D. M. Pfund, S. L. Wallen, M. Newville, E. A. Stern, and Y. Ma, J. Chem. Phys. 105, 2161 (1996).

${ }^{13}$ T. M. Seward, C. M. B. Henderson, J. M. Charnock, and B. R. Dobson, Geochim. Cosmochim. Acta 60, 2273 (1996).

${ }^{14}$ T. M. Seward, C. M. B. Henderson, J. M. Charnock, and T. Driesner, Geochim. Cosmochim. Acta 63, 2409 (1999).

${ }^{15}$ M. M. Hoffman, J. G. Darab, B. J. Palmer, and J. L. Fulton, J. Phys. Chem. 103, 8471 (1999).

${ }^{16}$ W. A. Bassett, A. J. Anderson, R. A. Mayanovic, and I-M. Chou, Chem. Geol. 167, 3 (2000).

${ }^{17}$ W. A. Bassett, A. J. Anderson, R. A. Mayanovic, and I-M. Chou, Z. Kristallogr. 215, 711 (2000).

${ }^{18}$ A. A. Chialvo and P. T. Cummings, in Advances in Chemical Physics, edited by I. Prigogine and S. A. Rice (Wiley, New York, 1999), Vol. 109, pp. 115-205.

${ }^{19}$ Th. Kowall, F. Foglia, L. Helm, and A. E. Merbach, J. Am. Chem. Soc. 117, 3790 (1995).

${ }^{20}$ R. E. Mesmer, W. L. Marshall, D. A. Palmer, J. M. Simonson, and H. F. Holmes, J. Solid State Chem. 17, 699 (1988).

${ }^{21}$ M. Maeda, T. Ito, M. Hiro, and G. Johansson, Z. Naturforsch. 51a, 63 (1996).

${ }^{22}$ G. Paschina, G. Piccaluga, G. Pinna, and M. Magini, J. Chem. Phys. 78, 5745 (1983).

${ }^{23}$ R. F. Kruh and C. L. Standley, Inorg. Chem. 1, 941 (1962).

${ }^{24}$ P. Dreier and P. Rabe, J. Phys. C 47, C8-809 (1986).

${ }^{25}$ P. L. Goggin, G. Johansson, M. Maeda, and H. Wakita, Acta Chem. Scand. A38, 625 (1984).

${ }^{26}$ P. Lagarde, A. Fontaine, D. Raoux, A. Sadoc, and P. Migliardo, J. Chem. Phys. 72, 3061 (1980).

${ }^{27}$ M. J. Apted, G. A. Waychunas, and G. E. Brown, Geochim. Cosmochim. Acta 49, 2081 (1985).

${ }^{28}$ J. R. Reitz, F. J. Milford, and R. W. Christy, Foundations of Electromagnetic Theory, 3rd ed. (Addison-Wesley, Reading, MA, 1979).

${ }^{29}$ Th. Kowall, F. Foglia, L. Helm, and A. E. Merbach, J. Am. Chem. Soc. 117, 3790 (1995).

${ }^{30}$ A. M. Couture and K. J. Laidler, Can. J. Chem. 34, 1209 (1956).

${ }^{31}$ L. G. Hepler, J. Phys. Chem. 61, 1426 (1957).

${ }^{32}$ S. F. A. Kettle, Physical Inorganic Chemistry: A Coordination Chemistry Approach (Spektrum Academic, Oxford, UK, 1996). 
${ }^{33}$ B. G. Kyle, Chemical and Process Thermodynamics, 3rd ed. (PrenticeHall, Upper Saddle River, NJ, 1999).

${ }^{34}$ J. O’M. Bockris and A. K. N. Reddy, Modern Electrochemistry (Plenum, New York, 1970), Vol. I.

${ }^{35}$ Y. E. Gorbaty and A. G. Kalinichev, J. Phys. Chem. 99, 5336 (1995).

${ }^{36}$ E. H. Oelkers and H. C. Helgeson, Science 261, 888 (1993).

${ }^{37}$ A. A. Chialvo, P. T. Cummings, and J. M. Simonson, J. Chem. Phys. 113, 8093 (2000)
${ }^{38}$ T. Driesner and P. T. Cummings, J. Chem. Phys. 111, 5141 (1999).

${ }^{39} \mathrm{~J}$. H. Weare (private communication).

${ }^{40}$ M. I. Lubin, E. J. Bylaska, and J. H. Weare, Chem. Phys. Lett. 322, 447 (2000).

${ }^{41}$ J. H. Weare, in Eleventh Annual V. M. Goldschmidt Conference, Abstract \#3871. LPI Contract No. 1088, Lunar and Planetary Institute, Houston (CD-ROM). 